\title{
Cultura organizativa de investigadores y entorno político y social
}

\author{
Joan Bellavista \\ Universitat de Barcelona. Spain \\ Tim Turpin \\ University of Wollongong. Australia
}

Stephen $\mathrm{H}_{\text {ill3 }}$

O ficina de la U N ESCO para la Ciencia y la Tecnología en el Sudeste asiàtico. Jakarta, Indonesia

Jesús M. de M iguel

Universitat de Barcelona. Spain

\section{Resumen}

La organización de la investigación se transforma de forma rápida y profunda en la mayoría de países. Las fronteras tradicionales de la ciencia se cuestionan por la influencia de la industria y el gobierno. Estas afectan a multitud de países y la investigación científica se lleva a cabo mediante diferentes imperativos cualitativos como respuesta a mercados diferentes de los que predominaron durante las décadas anteriores. A parece un espacio social donde se rediscuten las fronteras entre las culturas de investigación. Para muchos gobiernos, el impacto de los cambios del sistema científico en el desarrollo social y cultural se convierten en una prioridad. Las instituciones son más competitivas, más orientadas en lo comercial, más estratégicas, introduciendo prácticas y valores de gestión. En concreto se analizan los cambios que se operan mediante un análisis longitudinal de los casos de España y Australia.

Palabras clave: ciencia, tecnología, política, sociedad, cultura, organización.

\section{Abstract. Researchers organizational culture and their political and social environment}

The organization of research is changing fastly in most countries. Research traditional boundaries are beeing questioned by industry and government. The latter occurs in many countries, and scientific research is carried out as an answer to new markets. There is a social space where research cultures' boundaries are reviewed. For many governments, the impact of changes in the scientific system upon social and cultural development becomes a priority. Institutions are more competitive, commercially oriented, more strategy-involved, and acquiring management practices and values. In particular, the evolution of these changes are analysed for Spain and Australia.

Key words: science, technology, policy, society, culture, organization.

\section{Sumario}


La base teórica del presente artículo se basa en la idea de que la cultura tiene poder en la vida de los seres humanos, en el día a día de las instituciones y en las estructuras organizativas formales o complejas․ La cultura es también el producto del pensamiento y de las ideas humanas². Siguiendo a sociólogos clá sicos como Simmel, y contemporáneos como H abermas y Bourdieu, la cultura es el producto objetivo del pensamiento y de la acción subjetiva. D esde esa perspectiva aparece la tarea de explicar la dialéctica entre la cultura y las estructuras formales organizativas dentro de las cuales se encuentran 3 . La descripción del cambio en la investigación institucionalizada, descrita y contrastada con otras experiencias internacionales en el presente artículo, permite un contexto analítico para entender sociológicamente las relaciones entre las tendencias internacionales y las estructuras organizativas y culturales particulares ${ }^{4}$.

El presente análisis sociológico comparativo se basa en tres grupos de entrevistas en profundidad realizadas a investigadores e investigadoras, durante los años 1983, 1985 y 19915. Las entrevistas de 1983 se refieren a una muestra de veinte investigadores del área de Barcelona en un estudio financiado por la CIRIT6. Las de 1985 corresponden a una muestra de entrevistas en profundidad correspondientes a ochenta investigadores en C ataluña7. Las entrevistas de 1991 corresponden a treinta investigadores de la U niversitat de Barcel ona, realizadas para un estudio sobre la evaluación de la investigación de la CICYT8.

1. La hipótesis se discute en Jesús M. de M IGUEL (1979). El mito de la sociedad organizada. Barcelona: Península, 171 p.

2. Véase el recientelibro de Emilio LAM O DE ESPIn OSA, JoséM. GonZÁLEZ García, y Cristóbal T O RRES (1994). La sociología del conocimiento y de la ciencia. M adrid: Alianza, 632 p. También se puede consultar Esteban M EDIN A (1985). Conocimiento y sociología de la ciencia. M adrid: C entro de Investigaciones Sociológicas, $345 \mathrm{p}$.

3. Cristóbal Torres Albero (1994). Sociología política de la ciencia. M adrid: Centro de Investigaciones Sociológicas, 239 p.

4. Un estudio de interés para comparar con el caso español es el de Jacquel ine Fo RTES y Larissa ADIER LOMNITZ (1994). Becoming a Scientist in M exico: The Challange of Creating a Scientific Community in an Underdeveloped Country (U niversity Park, Pensilvania: T he Pennsylvania State U niversity Press, $225 \mathrm{p}$.

5. Una idea similar aparece en el ya clásico estudio de Joseph BEN-D AVID (1984). TheScienti t's Role in Soci ety: A Comparative Study. Chicago: The U niversity of Chicago Press, 209 p. Es una nueva edición (con una introducción adicional) de la de 1971.

6. El estudio de 1983 realizado por J. Bellavista, fue posible gracias a las ayudas a la investigación de la CIRIT, a quien se agradece la financiación.

7. Esas entrevistas son una muestra de las real izadas por J. Bellavista en el proyecto publicado en In STITUT d'ESTUdis Catalans (1990). La recerca científica i tecnològica a Catalunya. Barcel ona: G eneralitat de Catalunya, 3 vols. EI trabajo se realizó gracias a la financiación de la CIRIT y a la gestión del Institut d'Estudis Catalans.

8. Se agradece a la CICYT la financiación del proyecto de investigación del Plan Nacional PBS90-0160. Los resultados del conjunto del proyecto están publicados en J. BELLAVISTA, L. Escribano, M. Grabulós, C. Viladiu, E. Guardiola y C. I glesias (1993). Política científica y tecnológica: Evaluación de la investigación en la U nivers tat de Barcelona (Barcelona: Publicacions de la Universitat de Barcelona. Ver también, J. BeLLAVISTA, L. ESCRIBANo, M. Grabulós, E. Guardiola, C. Viladiu y C. I glesias (1992). «Focussing the manage- 
Los resultados de 1983 y 1985 se mezclan y se comparan con los de 1991. Se realiza aś un estudio comparativo y evolutivo del estado de la investigación en aspectos de organización, financiación, formación, intercambio, evaluación, productividad y políticas de investigación del G obierno central, del G obierno de la Generalitat de C atalunya y de la U nión Europea. Los resultados se comparan posteriormente con los antípodas, la experiencia evolutiva del caso australiano, aprovechando el análisis realizado durante dos años en el Centre for Research Policy de la U niversidad de W ollongong, en Australia9.

D esde la sociología se observa que la investigación y las universidades cambian 10 . Todo ello se produce dentro de un contexto general de cambio social que trata de equilibrar los procesos de desarrollo económico con las desigualdades sociales 11 . Tanto en España como en Australia, la investigación se va sistematizando. Las instituciones se convierten en más competitivas, más orientadas en lo comercial y más estratégicas. Introducen prácticas y valores de gestión. Al mismo tiempo, esos cambios estructurales llevan a tensiones entre las estructuras del pasado - los departamentos universitarios y las unidades de investigación basados en disciplinas científicas- y las nuevas estructuras de investigación que son centros o institutos de investigación, aś como redes de cooperación investigadora. Aunque los recursos públicos son cada vez menos esenciales, los gobiernos piden una mayor responsabilidad en el gasto. La política de investigación elige como objetivo las fronteras entre sectores y entre disciplinas ${ }^{12}$.

\section{Ciencia, tecnología, organización}

Los investigadores y las investigadoras del siglo xx se han tomado en serio el dictum de A.N . W hitehead de que «una ciencia que titubea en olvidar a sus fun-

ment and evaluation of the $R \& D$ resources at the University of Barcelona». $R \& D$ Evaluation N ewsletter 92.4, p. 7-10. También en J. Bellavista, L. Escriban O, M. Grabulós, E. GUARDIOLA, C. I GLESIAS, y C. VILADIU (1992). «La organización de la investigación en la universidad». Revista Española de Investigaciones Sociológicas, 59, p. 301-309.

9. Se agradece al M inisterio de Educación y Ciencia la financiación de la estancia de J. Bellavista en el Centre for Research Policy, centro especial de investigación del Australian Research Council, dedicado al estudio de las políticas científicas y tecnológicas y sus implicaciones sociales, lo cual ha hecho posible esta colaboración.

10. Un análisis organizacional puede verse en Jesús M. DE M IGUEL, Anatomía de una universidad: Para una Sociología de las O rganizaciones. Barcelona: D opesa, 1978, 139 p.

11. Se puede consultar el reciente estudio de la Fundación Foessa: Q uinto informe sociológico sobre la situación social en España: Sociedad para todos en el año 2000. M adrid: Fundación Foessa, 1994, 2.313 p. 2 volúmenes; sobre todo el capitulo primero, Jesús M. DE M IGU EL: «La España del cambio» (p. 1-144).

12. Joan Bellavista (D epartament de Sociologia, U niversitat de Barcel ona); Stephen H ill (director de la O ficina de la U N ESC O para la Ciencia y la Tecnología en el Sudeste asiático. Jakarta. Indonesia); Tim Turpin (director C entre for Research Policy, University of Wollongong); Jesús M . de M iguel (D epartament de Sociologia, U niversitat de Barcelona). 
dadores está perdida»13. La organización de la investigación en universidades e instituciones públicas de investigación sufre transformaciones rápidas e importantes en los últimos años en la mayoría de los países. Aunque no se puede hablar de un proceso cerrado, sí se puede observar que recientemente la ciencia se reorganiza en fórmulas nuevas. Las fronteras tradicionales entre disciplinas se cuestionan a través de un nuevo enfoque multidisciplinario en problemas de investigación aplicada. Éstos vienen definidos por instituciones fuera de las disciplinas científicas, como la industria y el gobierno14.

En ese contexto multidisciplinario, los centros de investigación emergen como entidades estructurales de investigación que trascienden las fronteras científicas que previamente se mantenían con fuerza en los departamentos y facultades (H ill y Turpin, 1993)15. En estas nuevas fórmulas que afectan a multitud de países, la investigación científica se lleva a cabo mediante imperativos cual itativos diversos. Es la respuesta a mercados que son bastante diferentes de los que predominaron durante los años setenta y ochenta ( $Z$ iman, 1991, LaPidus y otros, 1993; H ill y Turpin, 1994)16. Estas nuevas estructuras científicas, y los imperativos que las conducen, alimentan el espacio social donde se vuelven a discutir las fronteras entre las culturas de investigación. Todo apunta a que ese debate continuará en el futuro.

Las características son internacionales, pero se observan variantes locales. Gran Bretaña, por ejemplo, Ileva a cabo lo que se ha dado en Ilamar una revolución de la «cultura empresarial» que está dejando huella en sus universidades e instituciones científicas (Aberchromby, 1991; N ewby, 1992)17. En Europa, los nuevos acuerdos entre universidades, así como entre universidades e industria, transforman la investigación universitaria. Puntualizan así en áreas y disciplinas de investigación que poseen el potencial de dominar a las instituciones en vez de ser dominadas por éstas. Incluso en Asia el sistema científico - en China por ejemplo- toma una nueva apariencia empresarial. La experiencia asiática se caracteriza además por el conocimiento político de demandas globales de consistencia en materia de

13. Curiosamente este lema - aparentemente revolucionario - es adoptado por la sociología funcionalista, y propuesto como inicio de la famosa obra de Robert K. M erton sobre Teoría y estructura sociales. La contribución de M erton es básica para entender el desarrollo de la sociología de la ciencia en el mundo durante estas últimas décadas.

14. Un ejemplo aparece en Jesús M. DE M IGUEL (1991). «l+D en sociología». Revista Española del nvestigaciones Sociológicas, 56, p. 167-196.

15. S. H ILL Y T. TURPIN (1993). «The formation of research centres in the Australian research system». Science and Technology Policy, 6 (5).

16. J. ZIM AN . (1991). «Academic science as a system of markets». H igher Education Q uarterly, 45 (1); J. B. LAPID us, P. D. SYverso N, y S. R. W ELCH (1993). Postgraduate research training in the U nited States. Comunicación presentada en el U S C ouncil for G raduate Schools for the OECD Workshop on Research Training, Amsterdam; S. H ILL y T. TURPIN (1995). «T he clashing of academic symbols». Science as Culture.

17. H. N EW BY (1992). «N ew arrangements for the organisation and funding of research in the United Kingdom: An ESRC perspective». Centre for Research Policy, Working Paper, 5. 
propiedad intelectual, tanto en sus prácticas como en los acuerdos y en la política a seguir ${ }^{18}$.

Para muchos gobiernos la valoración e interpretación del impacto de los cambios del sistema científico en el desarrollo social y cultural se convierte en una prioridad. En España los cambios estructurales tienen un impacto en otras instituciones como las educativas, el empleo, sectores industriales, y en la forma en que los departamentos gubernamentales perciben la investi gación y el desarrollo. Pero, al mismo tiempo, esas instituciones permiten el entorno social en el que las nuevas estructuras de investigación muestran su impacto cultural y social.

La base teórica de la presente investigación parte de la idea de que la cultura tiene poder en la vida de los seres humanos, en la vida de las instituciones y en la de las estructuras organizativas formales. La cultura es también el producto del pensamiento y de las ideas humanas. Siguiendo a Simmel, y a otros autores más contemporáneos como $\mathrm{H}$ abermas y Bourdieu, la cultura es el producto objetivo del pensamiento y de la acción subjetiva. En consecuencia, no es especialmente importante que la cultura y la estructura sean construidas socialmente, sino que el tema fundamental está en el conflicto existente entre las dos. $\mathrm{H}$ abermas argumenta que la sociedad está cada vez más sistematizada. Las actividades de investigación y la producción y transferencia de conocimiento científico que se realiza en las universidades e instituciones de investigación, se sistematiza durante los años ochenta y sigue en los noventa (H ill y Turpin, 1994)19.

D esde esa perspectiva es importante explicar la dialéctica entre la cultura y las estructuras formales organizativas dentro de las cuales se encuentran20. En la teoría social, la tecnología se concibe a menudo como un producto social (artefacto cultural) o como una delimitación para los productos del esfuerzo humano (tiranía de la tecnología)21. La tecnología se usa con menos frecuen-

18. D. RUICHAN (1993). «Policy and legislation on the comercialisation of science and technology in China». Comunicación presentada en la Science and Technology for Economic and Social Progress PhD Symposium, Wollongong, Australia.

19. S. H ILL Y T. TURPIN (1995). «T he clashing of academic symbols». Science as Culture.

20. Una obra básica es la de C harles Perrow (1990). Sociología de las organizaciones. M adrid: M cG raw-H ill, 369 p. Es importante también consultar M auro F. G ULILÉn (1994). M odels of $\mathrm{M}$ anagement: Work, Authority, and O rganization in a Comparative Perspective. Chicago: The University of Chicago Press, 361 p. Para el caso español es imprescindible leer Alejandro N Ieto (1984). La organización del desgobierno. Barcelona: Ariel, 192 p. El análisis de un caso concreto se puede ver en Jesús M. DE M IGUel y M anuel M ART ín (1995). «Matar al padre: un modelo de reconstrucción del conflicto social desde la sociología de las organizaciones». Papers. Revista de Sociologia. O tros ejemplos internacional es aparecen en el libro de Arthur L. Stinch combe (1996). Stratification and Organization: Sel ected Papers (C ambridge: Cambridge University Press. Ver también, a nivel teórico: Josep A. Ro d Ríguez y M auro F. G UILLÉN (1992). «O rganizaciones y profesiones en la sociedad contemporánea». Revista E spañola de Investigaciones Sociológi cas, 59, p. 9-18; y todo ese número extraordinario sobre O rganizaciones y profesiones.

21. Una discusión reciente sobre el tema se puede encontrar en B. Pfaffen berger (1992). «Social Anthropology of technology». Annual Review of Anthropology, 21. 
cia para explorar la dialéctica entre cultura y sociedad como un problema. Simmel describe la tecnología como uno de los muchos órdenes culturales que emergen de la objetivización del pensamiento subjetivo22. H ill (1989), por su parte, sigue el camino de los desarrollos tecnológicos para observar el texto cultural que sólo se puede entender exponiendo la dialéctica entre cultura y sociedad. Las estructuras de investigación que se crean y se transforman en la década actual se pueden entender también como texto cultural. Pero ese texto sólo se puede comprender si tanto las formas organizativas - artefactos objetivos - como el imperativo cultural - significado subjetivo- se exploran en su punto de intersección. En particular, ese proceso selleva a cabo durante la producción sistemática de conocimiento en las instituciones de investigación.

La descripción del cambio en la investigación institucionalizada, descrita y contrastada con otras experiencias internacionales en el presente artículo, permite un contexto analítico para explorar las relaciones entre las tendencias internacionales y las estructuras organizativas y culturales particulares23. Ese contexto también es importante porque la política de investigación tiene que ver con factores de desarrollo social, económico y cultural. Sin embargo, hay que aceptar que un proyecto analítico de esta natural eza lleva también a plantear preguntas acerca de la cultura, las formas organizativas y la sociedad.

\section{Análisis comparativo longitudinal}

El análisis comparativo se basa en tres grupos de entrevistas en profundidad a investigadores e investigadoras realizadas en 1983, 1985 y 1991. Las entrevistas de 1983 se realizan a una serie de veinte investigadores del área de Barcelona en un estudio financiado por la CIRIT. Las de 1985 corresponden a una muestra de entrevistas en profundidad correspondientes a ochenta investigadores trabajando en Cataluña. Las entrevistas de 1991 corresponden a treinta investigadores de la U niversitat de Barcel ona, realizadas durante un proyecto sobre evaluación de la investigación de la CICYT. Los resultados de 1983 y 1985 se mezclan y se comparan con los de 1991 realizando un estudio comparativo y evolutivo del estado de la investigación en aspectos de organización, financiación, formación, intercambio, evaluación, productividad y políticas de investigación del gobierno central, del gobierno de la $\mathrm{G}$ eneralitat de Catalunya y de la U nión Europea. Los resultados se comparan posteriormente con la experiencia evolutiva del caso australiano, aprovechando el análisis realizado durante dos años en el Centre for Research Policy de la U niversidad de Wollongong en Australia.

22. G. SIM M EL (1918). «The conflict in modern culture». En George SIM M EL, On Individuality and Social Forms, D.N. Levine (ed.). Chicago: The U niversity of C hicago Press, 1971.

23. Un artículo lleno de ideas es el de Arthur L. St In Ch com Be (1990). «U niversity administration of research space and teaching loads: $M$ anagers who do not know what their workers are doing», p. 312-340, en su libro Information and Organizations. Berkeley: U niversity of California Press, $391 \mathrm{p}$. 
En el primer período analizado correspondiente a la primera mitad de los ochenta, las entrevistas realizadas muestran que la organización del trabajo viene marcada por la falta de personal administrativo, de gestión, de mantenimiento y técnico. Representa un comentario común a todas las entrevistas la crítica a la política de personal en el mundo investigador. Estas limitaciones conllevan un modelo de organización en el que el investigador y la investigadora individual o su grupo llevan a cabo tareas que no consideran formen parte de su trabajo, pues la resolución de los problemas administrativos y técnicos los resuelven los mismos investigadores con la consiguiente pérdida de tiempo para investigar. Esta falta de personal implica también que no se completan las necesidades de trabajo organizado del equipo de investigación. N o se habla propiamente de planificación del trabajo pero sí de organización del trabajo cuando existe un grupo de investigación en funcionamiento que tiene que tomar decisiones. Por otra parte, consideran que el trabajo administrativo que tienen que realizar demuestra la falta de flexibilidad del sistema ciencia-tecnología que afecta al modelo de organización dentro del equipo de investigación y negativamente a su propia flexibilidad en el trabajo. Se insiste en el problema expresado no tan sólo en un sentido de racionalidad, sino también en términos de agotamiento y desesperación; en muchos casos se consideran víctimas del trabajo burocrático que tienen que realizar pero también del entorno y la cultura burocrática existente en el sistema universitario y en términos más generales del sistema ciencia-tecnología. La cultura investigadora muestra una frontera con la cultura burocrático-administrativa e insiste en definir esta frontera para poder definir su modelo de organización. Sin embargo, son también conscientes de que este problema no se resolverá fácilmente y aceptan la resignación de realizar esos trabajos administrativos, con el fin de conseguir financiación para investigar.

La preocupación por la resolución de tareas administrativas y burocráticas no parece haber desaparecido en los años noventa, y la falta de un mayor apoyo para la gestión de los problemas cotidianos continúa siendo comentada con profusión. Sin embargo, el balance de preocupaciones dentro del contexto del uso del tiempo laboral parece trasladarse hacia consideraciones sobre el exceso de docencia y el trabajo que conlleva de preparación y evaluación de los alumnos y las alumnas. El personal que dedica muchos esfuerzos a la investigación es especialmente sensible a este tema, puesto que considera que la actividad investigadora resta una parte importante de las horas de trabajo y capacidad de organizar su actividad docente. Su actividad investigadora implica también más trabajo administrativo y de gestión adicional además de trabajo técnico en el laboratorio, todo lo cual lo separa aún más del número de horas dedicadas por el académico no investigador.

D entro de este modelo el personal que dedica parte de su tiempo a investigar explica como entra en un círculo de aumento progresivo del trabajo que se produce como consecuencia de esa actividad y ello le separa conceptual y prácticamente de la cultura del personal que se dedica exclusivamente a docencia. El incremento de la actividad investigadora en los últimos años lleva a que 
este tema tome cuerpo precisamente en el segundo análisis correspondiente a las entrevistas de 199124. Es interesante observar cómo a pesar de la mejora técnica de los sistemas de gestión universitaria, el incremento del volumen y la complejidad de las actividades es paralelo, con lo que continúan apareciendo problemas de gestión argumentando la necesidad de simplificar este mode lo de gestión. Es también importante observar el cambio que representa la entrada de los investigadores y las investigadoras en la cultura organizativa y de gestión como parte integrante del trabajo cotidiano y como parte de los elementos de discusión y cultura investigadora, todo ello dentro de este modelo incremental de actividad y complejidad dela organización del trabajo.

Paralelamente, el aumento de la cultura investigadora lleva también a conflictos con la disposición de la jerarquía docente. A menudo se provocan disfunciones entre las jerarquías docente e investigadora, la primera con un proceso legal institucional y la segunda a través de los resultados y prestigio investigador y consenso del grupo. Esta característica se confirma en el segundo análisis debido al incremento de la actividad investigadora respecto a la etapa anterior. D entro de la jerarquía investigadora aparece la función de coordinador - poco perceptible en el análisis anterior- que suele coincidir con el líder del grupo que habitual mente lleva a cabo la gestión y búsqueda de recursos para investigación. Es importante destacar el consenso sobre el liderazgo en el grupo cuando éste se ha formado por intereses comunes en un tema de investigación, en especial en su etapa ascendente.

En los años ochenta, el funcionamiento de los grupos de investigación requiere buenas relaci ones profesi onales y también personales. Es importante la buena comunicación entre los miembros integrantes del grupo. Los conflictos internos se atribuyen a la lucha por conseguir los pocos recursos existentes y liderar proyectos dentro de factores relacionados con la competencia por el prestigio y reconocimiento, la competencia por conseguir plazas fijas, y una situación general de sueldos bajos que no incentiva y provoca conflictos internos por la falta de expectativas. Según los investigadores y las investigadoras, ello contrasta con el mundo industrial y aparece el «fantasma» de los sueldos de la industria, aunque en realidad se conoce poco la situación real de éstos. Sin embargo, la competencia se considera positiva en otros casos, y un factor de mejoramiento de la calidad y productividad. Los factores de convivencia se colocan en los años noventa al mismo nivel de los factores profesionales, y se especifica que no se limitan a las horas de trabajo sino también a las relaciones personales que se establecen fuera de ese contexto. El efecto principal se produce cuando existe competencia y problemas de liderazgo dentro de un grupo en funcionamiento; los problemas profesionales y personales se mezclan dentro de una mecánica de posicionamiento individual y no de grupo. Sin embargo, es interesante observar que aparecen pocos comentarios sobre las relaciones personales en esta segunda época. Aunque no se puede afirmar que

24. Ver Joan Bellavista y otros (1993). Política científica y tecnológica: I +D en la U niversitat de Barcelona. Barcelona: Publicacions de la U niversitat de Barcelona, 331 p. 
el problema haya disminuido o desaparecido, lo que sí se puede notar es la gran concentración en los problemas de tipo profesional.

En la primera época se denota un factor de esperanza en la mejora de la situación futura basada en que esperan cambios positivos para la situación de la investigación, recordando siempre la pobre situación actual. Esta esperanza se mueve hacia el terreno de la organización del trabajo que creen se verá afectada directamente por estas mejoras futuras posibles. En al gunos casos manifiestan que ya han empezado algunos cambios concretos, rompiendo así con los mal os hábitos organizativos recogidos durante la historia pasada del grupo y del entorno investigador en general. Se considera que la disponibilidad de más recursos y material obligará a los grupos a cambiar sus fórmulas organizativas, que a veces se citan como simplemente inexistentes, para poder gestionar un volumen de recursos que no puede funcionar aislado.

A ello se le añade la visión de que los investigadores y las investi gadores individuales irán desapareciendo en favor de los grupos de investigación en un contexto de estructuras y organización nuevas. A parece una cierta insistencia en la necesidad del trabajo en equipo y parece observarse un proceso de concienciación sobre ese modelo grupal. Se insiste en la necesidad de una definición más precisa de las tareas y responsabilidades del personal para completar un modelo de organización del trabajo en equipos dinámicos y coordinados, y con una división precisa del trabajo entre sus miembros. La responsabilidad en el trabajo definida dentro de la estructura de los grupos es un tema sobre el que no se incluye una visión evolutiva. Se define como un factor más independiente del contexto histórico, que no tiene porqué evolucionar en el futuro. Empiezan a surgir comentarios sobre la necesidad de profesi onales en gestión de la investigación que se dedicarían a resolver parte de los problemas que corresponden al entorno del trabajo de investigación, y que creen no forman parte de su contrato. En ese caso se centran en la resolución de problemas administrativos simples. Parece existir la creencia de que el modelo organizativo al que habría que tender pasa por un sistema flexible, de comunicación ágil, interacción múltiple, más profesionalizado en la gestión, y con objetivos mejor definidos.

En la segunda etapa se propone un modelo de organización basado en el fomento de la interdisciplinariedad de los grupos o la agrupación multidisciplinaria de unidades diferentes, con líderes de los grupos actuando como investigadores principal es de proyectos o líneas de investigación con una idea crecientemente internacional, y una tendencia decreciente del trabajo individual. La identificación que los investigadores y las investigadoras tienen con su grupo se formula de una manera espontánea y no sigue pautas institucionalizadas de la estructura universitaria. Sin embargo, es un proceso dinámico de creación, identificación, separación y desaparición de grupos que interaccionan con diferentes grados de jerarquización de la organización del trabajo. Se comenta la necesidad de una gestión más dinámica y flexible acorde con las necesidades específicas de la gestión de la investigación y se pide un control a posteriori del gasto realizado en las tareas de investigación de los grupos, 
con menos intervención durante el proceso, puesto que se asegura va en contra de la flexibilidad y productividad del trabajo.

La necesidad de profesionales en gestión de la investigación se discute con mayor intensidad que en la etapa anterior y se le atribuye un carácter más amplio. N o se limita a la resolución de problemas administrativos puntuales, sino a la promoción de proyectos y programas, relaciones científicas internacionales y contratos. Forma parte de un modelo de gestión que debe resolver problemas más sofisticados en el contexto del aumento de la actividad investigadora y su internacionalización. Visto dentro del grupo, la mecánica organizativa considera la planificación como una utilidad parcial que no suele ser imprescindible para el desarrollo de los proyectos, sino más dedicada a la consecución de unos objetivos que permitan una financiación futura. El modelo de planificación, gestión y organización se refiere a la interacción entre la institución burocrática o financiera de la investigación y su grupo de investigación.

En los últimos años continúa existiendo una incertidumbre relativa a la continuidad del proceso de apoyo a la investigación. Ello se advierte como fundamental no en términos incrementalistas simples, sino como un proceso de acercamiento y normalización del estatus y de la cultura investigadora en el contexto europeo e internacional. Esta incertidumbre contrasta con la del análisis anterior, donde las esperanzas de normalización eran menores. 0 tra característica diferenciable es la mejor definición de los problemas en e/ trabajo de investigación. D e definiciones más generales se pasa a una caracterización más precisa de los temas. En concreto los problemas de espacio y entorno aparecen destacados sobre otro tipo de problemas, tanto por la explicación de su importancia como por el orden definido del peso específico del problema en un orden de prioridades de problemas a resolver. También por la explicación encadenada del problema respecto a otros problemas existentes, y la interdependencia existente que se establece con más profundidad que en el período anterior.

La falta de recursos para investigar es un tema común a todos los entrevistados y las entrevistadas de la década anterior. Se establecen comparaciones continuas con otros países que disponen de mayor capacidad. Algunos y al gunas afirman disponer de unos mínimos de subsistencia, otros aseguran estar por debajo de ese nivel. Estos comentarios son generalizados en todo tipo de áreas de investigación y aparecen tanto en las áreas donde se necesitan muchos recursos, como en las áreas que requieren pocos recursos, con lo que parece ser una limitación fundamental para el desarrollo de la investigación en esta época. Las referencias a la falta de infraestructura son frecuentes, aunque también existen autocríticas sobre el mal uso y la fal ta de rendimiento de la infraestructura existente. Se insiste en que un aumento de presupuestos debe ir acompañado de un cambio en las estructuras organizativasy de gestión, condición indispensable para que los recursos sean real mente aprovechados. Los problemas de financiación afectan a la continuidad del trabajo de investigación. Ello lleva a discutir la necesidad de disponer de unos presupuetos mínimos anuales para asegurar esa continuidad. Son pocos los entrevistados y las entrevistadas que refieren 
una situación de recursos suficientes 0 aceptables. Parece haber unos pocos grupos que aisladamente disponen de los recursos de que nadie dispone, en un modelo donde existe una base extensa con unos recursos limitados o inexistentes, y una élite que sigue un trabajo de investigación continuo y sabe encontrar los recursos necesarios.

En la década actual, la financiación representa un condicionante dentro del proceso y desarrollo de la investigación. Pero a diferencia de la etapa anterior se observa una actitud positiva respecto a las posi bilidades de conseguirla. El conocimiento de los canales de financiación, aunque mejor, aún está poco desarrollado. Aseguran estar en un momento de mejora importante del acceso a la información sobre financiación que puede tener efectos sobre un futuro inmediato. Lo que sí se ha desarrollado es la diversificación y el conocimiento de la discusión sobre los temas de investigación. En la etapa anterior existía un comentario bastante común que se centraba en la falta de financiación. En la etapa siguiente se observan comentarios y discusión sobre el sistema, interés, prestigio, calidad, volumen, distribución, localización y resultados de la financiación. En bastantes casos la financiación ha dejado de ser un problema fundamental, y el tiempo y la organización son los verdaderos quebraderos de cabeza. Los problemas aparecen en los grupos no consolidados y sus dificultades para conseguir financiación. Se pide una implicación de la propia institución en la promoción y búsqueda de financiación.

Se observa, pues, en la primera etapa, que en general existe una cultura investigadora que contiene el concepto de la falta de recursos. Esta posición se denota en la expresión del sentimiento de frustración de muchos investigadores e investigadoras que no permite traspasar esta frontera conceptual de la cultura de investigación sin recursos, que a veces incluso se traduce en una subutilización de los recursos existentes. La hipótesis que barajan parece corresponder a una cultura investigadora con recursos, donde el desarrollo paralelo de los mecanismos de control de los recursos lleva a una mejor utilización del material de éstos; o sea una correlación positiva entre disponibilidad de recursos y capacidad de utilización. Aunque parcialmente, en la segunda etapa se denota un proceso de mejora y racionalización de la utilización de la infraestructura y recursos que tiende hacia la demostración de esta hipótesis. Sin embargo, se requiere realizar más investigación sobre este particular para avanzar en la comprensión del proceso.

Las relaciones entre la universidad y la empresa han tenido cierta importancia en las dos épocas examinadas, aunque los temas comentados puedan variar. En la primera época analizada, las relaciones que se establecen son de muchas clases. No se limitan a relaciones contractuales, sino también de intercambio de datos y documentación. Los aspectos positivos que aparecen en las relaciones universidad-empresa se refieren a la necesidad de la existencia de estas relaciones, tanto por factores de utilidad como de interés. A pesar de que las relaciones son aún poco abundantes y escasamente duraderas, existe una actitud positiva para abrir canales de comunicación y establecer relaciones futuras. Cuando aparecen posibilidades de relación, normalmente se intenta 
la interacción. Son pocos los que se niegan a establecer al gún tipo de relaciones por principios de la definición del trabajo de investigación. Lo que se observa es una relación entre investigación fundamental y actitud menos positiva de relacionarse con la industria por miedo a perder poder de decisión sobre las líneas o enfoques en sus investigaciones. La actitud es claramente positiva en los investigadores y en las investigadoras en trabajos más aplicados. C reen que estas relaciones aumentarán en el futuro, como fruto del interés de las dos partes. Existe la opinión de que se está cambiando la imagen tradicional de negarse a contactar con la industria - antes era mal visto diceny parece que en esta época ya ha empezado a generalizarse un cambio cultural de apertura de la investigación pública hacia la empresa y el exterior en general.

Como aspectos limitativos de esta relación se observa la existencia de una separación cultural entre el mundo de la investigación pública y la empresa privada, especialmente en la primera época. Algunos creen que la industria desconoce el potencial de la investigación pública, que la industria desconfía de la capacidad de la universidad para resolver sus problemas, y requiere los resultados más rápidos de lo que permite un proceso de investigación promedio. Se citan dificultades legal es para lograr un buen entendimiento. Aparecen experiencias negativas que muestran las dificultades de superar las diferencias entre estos dos mundos. Se observa la falta de preocupación por buscar los ben eficios que podría aportar la relación, aunque existe la opinión de que la desconfianza viene en muchos casos del desconocimiento, y que no puede romperse esta situación hasta intentar algún tipo de relación buscando el beneficio mutuo. D e todas maneras, parece observarse un acercamiento de posturas y un descenso de la desconfianza tradicional. La incapacidad de invertir a medio y largo plazo y la dimensión reducida de la empresa media se consideran factores limitativos.

A veces existe un interés escondido de la relación cuyo objetivo está más enfocado a la justificación de acciones y a la obtención de permisos que a la consecución de resultados concretos para su uso posterior. En muchos casos descalifican estas relaciones como investigación, definiéndolas como la resolución de problemas concretos y aplicaciones parciales en el sistema productivo que no requieren un proceso que siga las pautas de lo que ellos consideran investigación. Además se encuentran con el desconocimiento de la utilidad 0 aplicación final de su trabajo, que suele terminar antes de la aplicación final. Se comenta la necesidad de la intervención de la Administración para establecer las medidas necesarias que promocionen estas relaciones, y consideran escasa hasta el momento. Este papel de mediador se define como un proceso técnico que lentamente irá rompiendo las fronteras culturales entre las dos partes.

En los años noventa se vuelve a comentar con insistencia el proceso de cambio conceptual y cultural de los investigadores y las investigadoras respecto a las relaciones con la empresa, con una actitud cada vez más receptiva. Aceptan que en muchos casos la relación no sirve solamente para fines prácticos de desarrollo del acuerdo, sino que también proporciona un conocimiento 
y una experiencia nueva sobre el modelo de organización y trabajo empresarial. O bservan una predisposición mayor para mostrar su potencial y ofrecer servicios concretos. El factor económico de financiar investigación es determinante y característico de esta época, disponiendo de ingresos que servirán para el desarrollo de la investigación en el departamento. También es característico de esta época la entrada en la cultura económica y organizativa empresarial para mejorar su capacidad de gestión y negociación de contratos, y organización para cumplir los plazos de entrega de los trabajos.

Las críticas se centran en la calidad y definición del trabajo fruto de contratos. Existe una dicotomía entre definiciones sobre trabajo de investigación y «simple» servicio junto a la falta de continuidad. 0 tro grupo de críticas va dirigido a la definición de los intereses que se esconden detrás de la formulación de los contratos. Un ejemplo es el sueldo adicional que se puede obtener, que, aunque está dentro de la legalidad, se critica por considerar que puede ir en contra de la calidad e interés por el nuevo conocimiento y la prestación de servicios a bajo coste para la empresa. En muchos casos se acepta que ni tan solo existe un proceso de transferencia de conocimiento o tecnología. Sin embargo, estos comentarios suel en proceder de investigadores sin experiencias contractuales con la empresa, que trabajan en investigación de difícil aplicación industrial 0 en los que han tenido experiencias muy negativas. En ese sentido, se observan posturas radicales en un grupo cada vez más reducido de contrarios a establecer contratos. El proceso de apertura ya observado continúa evolucionando, aunque se mantiene dentro de una actitud positiva, una necesidad de rediscutir el papel, los objetivos y la ética de las relaciones dentro del contexto del marketing de la investigación.

Existe en la primera época una crítica constante a la formación universitaria recibida, y las deficiencias se definen tanto para el futuro investigador como profesional. La situación se cree que puede incluso empeorar con la masificación de las aulas. Esta formación se critica en especial cuando se enfoca como base de entrada para el trabajo investigador, tanto por la organización de la institución, dedicación y calidad del profesorado y deficiencias de metodología, como por la falta del material necesario para la formación investigadora. Sin embargo, también se exponen las excepciones citando a personas concretas como ejemplo de esas excepciones. Ello contrasta con la definición general de la importancia que dan a una formación que incluya elementos para preparar a los investigadores y a las investigadoras futuros. C reen que una buena formación para la investigación requiere transmitir elementos de imaginación, inventiva, creatividad, metodología enfocada hacia la investigación, critica, y menosimitación y respuestas estandarizadas.

La formación para la investigación en los años noventa se enfoca más hacia el seguimiento del tercer ciclo de la universidad y la tesis doctoral. El tema no se puede comparar directamente, puesto que el enfoque de las entrevistas va más en este estado de formación. Sin embargo, es importante observar como los investigadores y las investigadoras definen la formación para la investigación exclusivamente en ese estadio. El máximo rendimiento se aprovecha especial- 
mente cuando ese proceso se lleva a cabo dentro de un proyecto de investigación en el que se le permite estar incluido, puesto que la formación se extiende hacia factores de organización del trabajo en equipo; también cuando se llevan a cabo estancias de investigación en el extranjero. Aunque existen defensores decididos, aparece también una crítica hacia los cursos máster y de postgrado. Según al gunos entrevistados y entrevistadas, se realizan con baja calidad y poca organización, y a veces por razones más económicas que educativas. A pesar de las dificultades de comparar este punto, es interesante observar como en la década anterior la formación para la investigación se plantea de una manera general. Aparecen pocos comentarios sobre la formación de los ya graduados, y se insiste mucho más en las deficiencias de la carrera universitaria antes de la graduación.

Se considera que parte de la formación se adquiere con mucha lectura personal - autodidactismo- , visitas y posteriores contactos más estables con otros grupos, especialmente cuando éstos son extranjeros. El intercambio se considera una condición necesaria para estar al día, tanto si lo practican como si no. Es frecuente el comentario de la falta de medios para mantener contactos con grupos afines en otras instituciones a través de congresos y visitas, tanto para adquirir nuevo conocimiento como por el acceso a nueva metodología. Se critica que éste es uno de los temas con menos recursos disponibles, y que representa una limitación al desarrollo científico. En todo caso, no parece haber un intercambio verdadero de personal, sino una asistencia a reuniones, congresos y visitas cortas a centros, para temas puntuales. La discusión aparece en si las rela ciones y el intercambio científico deben seguir unas pautas de espontaneidad o deberían institucionalizarse para justificar la actividad y su financiación.

El intercambio de información, conocimientos y experiencia en los noventa se entiende en un primer grado como la asistencia a congresos y encuentros diversos para tratar temas específicos de investigación y para aprovechar los beneficios del contacto directo y personal. Aparece una riqueza comparativa de elementos y fórmulas de intercambio, que puede ser explicada por la aparición de nuevas modalidades de ayudas públicas a través de viajes, proyectos, etc. Las fórmulas que mejor resultan, sin embargo, parecen ser el mandar un miembro del propio grupo a trabajar temporalmente con otro grupo con el que se pretenden establecer relaciones futuras, y conseguir financiación para proyectos internacionales de investigación con grupos de otros países. Estas fórmulas de intercambio mantienen el ementos que, aunque más desarrollados en volumen y diversidad, se basan en los mismos parámetros de la década anterior. U n cambio más radical aparece en las nuevas fórmulas de intercambio inmediato a través del correo el ectróni co que al gunos entrevistados y entrevistadas apuntan como un nuevo modelo de intercambio instantáneo, que además permite la interconexión continua, no tan sólo con bases de datos de investigación, sino también con su propia red internacional de investigadores, con los que mantiene relaciones.

En la primera etapa analizada, parece haber bastante acuerdo en nombrar las publicaciones como el indicador de productividad del trabajo de investigación, 
aunque paralelamente no se cree que sea un indicador simple. La discusión reside en la valoración de la importancia y calidad de una publicación concreta, y a menudo se sugiere diferenciar una publicación nacional de otra publicación en una revista extranjera. En este punto existe un cierto simplismo en el uso del concepto de revista extranjera como sinónimo de calidad, en vez de usar el concepto de revista de prestigio internacional. En todo caso, citan las dificultades que tienen para conseguir publicar en revistas «extranjeras». Su preocupación reside en que no se valore exclusivamente la cantidad, sino también la calidad. Sin embargo, al gunos y al gunas creen imposible valorar la calidad, con lo que argumentan que quizás no haya más remedio que contar el núme ro simple de artículos publicados. La respuesta a este punto es el miedo a que se provoque una inflación de publicaciones de baja calidad por la mera nece sidad de tener un número simple de publicaciones realizadas. La productividad se relaciona además con la difusión que el conocimiento tenga dentro de la comunidad científica, con lo que aumenta el interés por publicar en revistas prestigiosas que sean leídas por el mayor número posible de científicos y científicas. Es interesante observar que no aparecen otros elementos destacados para valorar la productividad, y sólo pequeñas notas sobre la importancia de la utilidad de los resultados de las investigaciones.

Existe una discusión parecida sobre la problemática general de evaluar el trabajo de los investigadores y las investigadoras. Aunque aparecen declaraciones encontradas a favor y en contra de evaluar el trabajo científico, existen pocos argumentos que lo soporten, apareciendo como un tema nuevo, y una discusión con aún pocos elementos para argumentar. $\mathrm{H}$ ay defensores que se basan en el control del gasto público, y retractores que se basan en la imposibilidad de evaluar objetivamente, aunque las propuestas elaboradas son casi inexistentes. Existe la opinión de que para que la investigación pueda ser evaluada primero tiene que conseguir ser un tema social y políticamente importante, y las instituciones tienen que mejorar su sistema de gestión.

La necesidad de evaluar el trabajo de investigación parece entrar con fuerza en la segunda época, aunque se anota la necesidad de conseguir un cierto nivel de objetividad en las técnicas y los métodos comparativos de evaluación. La razón de esta actitud más decidida que en la etapa anterior parece estar en el contexto general de aceptar que cualquier organismo que financia tiene el derecho a evaluar el rendimiento de la inversión realizada, pero también para establecer criterios más objetivos de productividad en el trabajo. El indicador más aceptado para medir la productividad siguen siendo las publicaciones, a la que sigue toda una larga discusión sobre las limitaciones y los peligros que puede llevar inherentes. Sin embargo, a pesar de los problemas que puedan aparecer, continúa siendo el claro favorito. El concepto de «impacto» de la revista donde se publica el artículo según los estándares del Instituto de Información Científica de Filadelfia (ISI ) aparece en segundo lugar y entra en la cultura científica de la época actual, teniendo en cuenta que no aparece ningún comentario al respecto en las anteriores entrevistas. En este punto se sugie re que existe una confusión entre el factor de impacto de la revista y el número 
de citas que un autor recibe según la base de datos del ISI . En un tercer lugar está la aprobación de proyectos de investigación y las ponencias en congresos. Para terminar, los contratos de investigación, las patentes y las tesis dirigidas se citan en casos particulares.

Como técnicas de evaluación se sugiere también la continuidad del peer review o evaluación por colegas profesionales, como fórmula que funciona bien para la evaluación de proyectos. Algún comentario aislado sugiere el visiting group, que agrupa no sólo a científicos sino también a otros agentes sociales conocedores de la materia de la persona o grupo que hay que evaluar. En cualquier caso, es interesante destacar la gran riqueza comparativa de ese tema respecto a la etapa anterior y la aceptación de que hay que trabajar para la objetividad de los indicadores a través de un análisis conjunto de todos los indicadores útiles en un análisis evolutivo, siempre que existan mecanismos de defensa para el investigador y la investigadora respecto a los resultados de las evaluaciones. También aumenta la riqueza de discusión sobre los sesgos que pueden aparecer en los indicadores. La cantidad en contra de la calidad en las publicaciones es de habitual discusión; la propia calidad y la subjetividad que conlleva. Se discute la formación de redes de investigadores y la tendencia a la autoprotección de sus miembros. O tras críticas van dirigidas a los sesgos de las técnicas de evaluación que se basan en las bases de datos del ISI, como la comparación de áreas, sistema de citas, factor de impacto, etc. Para terminar, aparece una autoeval uación sobre la mejora generalizada de la productividad científica en los últimos años.

Cuando se habla de productividad y evaluación suele aparecer el tema del reconocimiento por el trabajo realizado y resultados. En los años ochenta existe una opinión generalizada de falta de reconocimi ento del trabajo que basan en su mala situación salarial respecto a otros profesionales aunque raramente plantean un posible cambio de trabajo. La libertad de trabajo y líneas de investigación parece ser un punto fundamental para no querer ir a trabajar a la industria u otra profesión con un mejor salario. La situación de insatisfacción parece que aumenta en muchos casos conforme pasan los años y la situación no suele cambiar para mejor. Las satisfacciones son más de tipo personal y suelen venir del trabajo de investigación cuando consiguen financiación para llevarlo a cabo, aunque ello no repercutirá sobre su salario. Para muchos investigadores éstos son verdaderos indicadores de la fal ta de reconocimiento. D entro de esta visión aparece pues un sistema disperso y poco competitivo, con una satisfacción que viene del trabajo personal y no del reconocimiento institucional.

Las comparaciones remunerativas con otras profesiones y otros colegas en el extranjero siguen apareciendo en los noventa, pero se centran más en las satisfacciones concretas que aporta el trabajo de investigación. Los factores de carácter personal y vocacional son fundamentales y la presentación de los resultados y el proceso para conseguirlos son fundamentales para su satisfacción. El acceso al nuevo conocimiento a través de un proceso creativo les estimula, aunque recuerdan la gran cantidad de trabajo rutinario que suele hacerse tam- 
bién. Ello no es óbice para que consideren que no están reconocidos socialmente en comparación con el trabajo que realizan, y en concreto por la imposibilidad de retener a los mejores estudiantes que normal mente no pueden seguir una carrera investigadora en la universidad. Los argumentos de creatividad, vocación, acceso al nuevo conocimiento y libertad para decidir sobre las líneas de investigación son los que usan para explicar el porqué tampoco quieren cambiar de trabajo. Aunque nos encontramos con una situación más competitiva en el sistema ciencia-tecnología, los argumentos sobre el reconocimiento no parecen diferir demasiado de los anteriores.

La discusión sobre las prioridades de investi gación es uno de los temas principales dentro de los estudios y análisis sobre políticas de investigación. En los años ochenta existe una opinión generalizada de que no existe un sistema de prioridades, y que ello viene de la falta de desarrollo de una planificación y objetivos en el sistema de ciencia-tecnología en España. Se habla de que con tan pocos recursos es imposible priorizar, y que sólo se puede priorizar un tema que disponga de una buena base y personas ya preparadas. Algunos insisten incluso en que la misma investigación no es prioritaria dentro de las prioridades de las políticas sectoriales de las administraciones. Algunos puntualizan, sin embargo, que parece notarse un nuevo interés en ambas administraciones sobre la política de investigación, aunque habrá que esperar para comprobar si es efectivamente cierto. Un problema general se observa en la falta de información sobre el estado actual de la investigación. Un estudio exhaustivo sobre el potencial de las líneas de investigación y recursos existentes, junto a un sistema de planificación para lograr unos objetivos concretos, se cita como previo al establecimiento de las prioridades.

La discusión entre la libre iniciativa de investigación versus la introducción de prioridades concretas es central en este tema. Existe bastante aceptación de la relación entre el trabajo científico y el aprovechamiento de los resultados por parte de la estructura productiva y la sociedad, lo cual puede llevar a establecer unas prioridades. Sin embargo, temen el proceso de decisión para el establecimiento de tales prioridades. Existe el temor de la fal ta de criterios claros en la distribución de los recursos y cambios debidos a factores también poco específicos - ni científicos ni económico-sociales- . Creen que los científicos y las científicas deberían ser consultados en estos temas. El posible dirigismo político sobre las prioridades aparece con frecuencia, lo cual relega al científico y a la científica a un segundo plano. El problema, pues, no parece tanto el hecho de las prioridades sino la manera como se lleve a cabo el proceso. En la vertiente de la libertad de investigación, se insiste en la imposibilidad de planificar la investigación teniendo en cuenta la dificultad de calcular las aplicaciones futuras del trabajo de investigación fundamental. La posición explica que sin esta investigación fundamental no habría una base para las aplicaciones futuras, y un sistema de priorización tenderá a ir en contra de la financiación de la investigación fundamental.

A pesar del incremento de presupuestos para investigación que se observa en la segunda etapa analizada, existe una conciencia sobre el concepto de recur- 
sos limitados para la investigación. Es a partir de ahí que aparece una defensa bastante generaliza sobre la necesidad de priorizar los recursos existentes en temas específicos. Los argumentos económico-sociales son centrales en defensa de la priorización. Se habla de la contribución de una investigación priorizada para las necesidades económicas, la industria, el desarrollo tecnológico y la sanidad. Sin embargo, las personas críticas temen una eliminación progresiva de los temas «no prioritarios» por falta de financiación futura y una tendencia de los investigadores y las investigadoras a cambiar artificialmente sus líneas de trabajo a causa de las posibilidades de financiarla, lo cual irá en contra de la motivación intelectual por un tema, y sólo a favor de su financiación potencial. La consecuencia de esta situación sería la desaparición de áreas importantes de la investigación básica. Aunque se acepta que ha habido un avance significativo sobre el concepto y el sistema de decisión sobre las prioridades, la discusión y crítica se enfoca hacia las prioridades específicas que determinan las administraciones, que se basan en un conocimiento poco exhaustivo de las necesi dades de investigación respecto a los obj etivos que se pretende conseguir. 0 tra crítica se refiere a la definición poco específica de las prioridades, y el peligro de financiar temas colaterales por la falta de esa definición más concisa y clara.

Las ideas sobre la política científica, se suelen enmarcar dentro del contexto de la política general española, especialmente en las entrevistas de 1983. Son abundantes los comentarios sobre el contexto político e ideológico del entorno de trabajo universitario. Sin embargo, se considera que la investigación sólo empieza a ser discutida limitadamente por parte de los políticos y las políticas. Cuando se habla de ello es porque empieza a haber una cierta moda aunque no se discute en profundidad, observándose el comentario general de la falta de una política científica por parte de las administraciones. Sin embargo, hay al gunas esperanzas de cambio para el futuro y esperan la aparición de las leyes de la ciencia, tanto la del Gobierno central como la de la G eneralitat, no tanto como una solución sino como un marco de referencia que dé mayor importancia a la investigación. La existencia de una política científica se define como una condición necesaria para el desarrollo de la investigación, y ello se conecta con la necesidad de ésta para el desarrollo de la creatividad, innovación, tecnología y el desarrollo socioeconómico. En concreto, existen múltiples observaciones sobre las diferencias de calidad de investigación con otros países, y la necesidad de reducir la dependencia científica, tecnológica y económica con el exterior. Es común la referencia a temas de investigación donde las diferencias con otros países son tan grandes que quizás ya se haya perdido el tren. La misión de una política de investigación sería promover la organización, el personal y los medios para la investigación, y a la vez requiere continuidad y objetivos definidos.

En general no se establecen grandes diferencias entre la política científica de la $\mathrm{G}$ eneralitat y la del Gobierno central. En realidad se conocen poco, y existe confusión sobre la propiedad de las acciones. Es habitual el comentario sobre la necesidad de la transferencia de competencias en materia de investigación, 
aunque se puntualiza que ello no tiene por qué mejorar la situación de la investigación. Parece así más un posicionamiento ideológico que práctico. Algunas personas creen que tendría que haber cambios radicales en la política de investigación, de lo contrario los problemas seguirían siendo los mismos pero con la Administración más cerca de casa. Se habla de una condición necesaria pero no suficiente. 0 tros están más convencidos de que no se puede evaluar la política de investigación de la G eneralitat hasta que existan competencias, y consecuentemente recursos administrados por ella. Para un tercer grupo de entrevistados, la coordinación entre administraciones: G eneralitat, diputaciones, G obierno central, parece la única fórmula de promover la investigación, de lo contrario siempre habría interpretaciones vagas sobre las competencias. Lo que no aparecen son comentarios sobre las comunidades europeas. En general no se entra en profundidad en las medidas concretas de política de investigación a excepción de la política de becas, con lo que parece haber un gran interés por el tema de la política de investigación pero sin entrar en profundidad. No se ha formado aún una cultura sobre el tema. Las críticas a las diversas administraciones son también poco profundas, y confusas en cuanto a la atribución sobre las acciones concretas.

La política científica de la década de los noventa se especifica dentro de las diferentes administraciones, y desaparecen buena parte de los conceptos genéricos sobre la política de investigación. Sobre la G eneralitat de C atalunya hay poca riqueza de comentarios sobre actuaciones específicas, y es más bien una toma de postura general sobre la existencia y la calidad de esta política. Se discute la falta de recursos conectada a la falta de transferencias en materia de investigación. Sin embargo, se critica la falta de prioridad de la investigación dentro de las políticas sectoriales de la $G$ eneralitat, aunque existe paralelamente una estimación sobre el inicio de una mejora de esta situación referido a la definición y rentabilización de las actividades. 0 tros comentarios van en el sentido de la importancia de los grupos de presión y las dificultades de realizar una política objetiva en una comunidad investigadora relativamente pequeña donde los especialistas y las especialistas se conocen personalmente.

La política de investigación del G obierno central se evalúa con más precisión. Se observa un incremento de recursos y métodos en la elaboración de la política de investigación. Existe una actitud positiva respecto a las mejoras de los últimos años, con la posibilidad de consolidación de grupos y aparición de otros nuevos. Los esfuerzos de definición de las políticas y el sistema de evaluación para la financiación de la investigación se valoran positivamente. Sin embargo, existe un cierto temor a que esta situación de mejora se rompa en cualquier momento. N o parece haber confianza en que estas mejoras duren mucho tiempo, lo cual parece relacionarse con que la opción por la investigación por parte del Gobierno central puede ser pasajera. En especial se habla de la necesidad de mejorar las infraestructuras y el volumen de becarios y becarias donde aún hay mucho por hacer. 0 tras críticas van dirigidas al mimetismo de esas políticas con las de otros países de la CEE, afirmando la inexistencia 
de especificidades por las necesidades económicas y social es específicas. La política de investigación de la Comunidad Europea es la menos conocida, aunque existe una actitud positiva a su existencia y una crítica a la definición casi exclusivamente aplicada de sus líneas de trabajo.

En una visión más general, es importante discutir la posición del científico y de la científica en la sociedad desde la perspectiva del propio científico o científica. En la primera etapa anal izada se sugiere un desconocimiento generalizado del trabajo de los investigadores y las investigadoras. Se parte de una idea de desconocimiento y falta de opinión. Cuando aparece, el científico o la científica no tiene un papel significativo en la escala social. Creen que la sociedad no está interesada en su trabajo ni en la importancia que la investigación pueda tener, lo cual está relacionado con la misma desconexión existente entre universidad y sociedad. Por otra parte, la idea del científico y de la científica como un trabajador más dentro del sistema productivo tampoco aparece, ya que la idea existente es poco concreta o simplemente desconocida. C reen que éste es un problema grave que afecta directamente a su posición dentro de la sociedad y a su falta de reconocimiento. La difusión y divulgación del trabajo científico podría ser un canal de mejora de su posición social, pero los pocos ejemplos existentes suelen ser de baja calidad y se caracterizan por su superficialidad a causa de la fal ta de preparación de los que divulgan el trabajo investigador en los medios de comunicación. Se considera que hace falta desarrollar un periodismo científico de calidad que no existe en el momento de las entrevistas. O tro canal podría ser un incremento de la formación científica de la sociedad, lo cual aproximaría a la sociedad hacia el conocimiento y comprensión de la ciencia. Todos están de acuerdo en que hay que poner remedio al problema, y más teniendo en cuenta la importancia creciente de los resultados del trabajo investigador en la sociedad. Sin embargo, no existe optimismo en cuanto a las posibilidades de cambiar esta situación.

En la segunda etapa no parece haber muchos cambios en la posición del científico y de la científica en la sociedad según el punto de vista de los propios investigadores e investigadoras. Su trabajo no es reconocido socialmente, como no lo ha sido nunca. La opinión generalizada es que prevalece la imagen de un trabajo básicamente docente con unas largas vacaciones. La idea es irreal y existe un sentimiento de frustración al no tener esperanza de que aparezcan grandes cambios en el futuro. Se habla de la necesidad de divulgar su trabajo con mayor asiduidad, en forums de gran audiencia, y en los medios de comunicación social. Sin embargo, se consideran incapaces de entender porqué es tan difícil romper esa imagen tan al ejada de la realidad. Exponen ejemplos prácticos como la reticencia de la empresa privada a contratar doctores universitarios, lo cual no facilita la divulgación de sus características en el mundo de la empresa. Los pocos cambios que observan han venido de una divulgación popular relativamente reciente del trabajo científico, y ello se toma como una situación posible, aunque difícil, de cambio futuro. Sin embargo, ello debería ir conectado con una mayor dedicación por parte del propio investigador en divulgar el valor de su trabajo en la sociedad. 
Las entrevistas de 1985 no contemplaban específicamente la opinión sobre temas éticos en la investigación. En consecuencia, sólo existen pequeños comentarios colaterales a través de otros temas. En las entrevistas de 1983 aparece la opinión de la inexistencia de responsabilidad del investigador por las consecuencias potencialmente negativas que pudiera tener el trabajo investigador, aunque aceptan la posibilidad de que existan efectos negativos. C reen que la ciencia no es peligrosa en sí misma, sino que la posible aplicación futura es la que puede convertirla en peligrosa. En otro orden se habla de la posibilidad de errores humanos del investigador y de la investigadora generadores de riesgo, los cuales se pueden encontrar en cualquier otro nivel profesional. En todo caso, la ausencia de intencionalidad demostraría la ausencia de responsabilidad. A principios de los años noventa, son casi inexistentes los casos de científicos y científicas entrevistados que acepten tener alguna responsabilidad sobre efectos negativos o de riesgo potenciales como consecuencia del trabajo o de los resultados de la investigación. En algunos casos la opinión se conecta con la inexistencia de riesgo a corto o largo plazo de su línea de investigación, pero suelen hablar de la inexistencia de responsabilidad del científico y de la científica en general. Argumentan la dificultad de conocer estos posibles efectos en la mayoría de los casos. Creen que estos problemas pueden aparecer en la fase de desarrollo y uso posterior, en la cual no suelen participar y está fuera de su control, y en consecuencia fuera de su responsabilidad. La responsabilidad se traslada, pues, a quien utiliza los resultados de la investigación, que puede ser una empresa, un uso político, o en todo caso se habla de una responsabilidad social compartida. Paralelamente, insinúan la necesidad de un control internacional sobre los países que permiten desarrollos peligrosos, y controles locales sobre actividades más localizadas. C reen en la existencia de una cultura de responsabilidad del trabajo concreto que realizan las científicas y los científicos, y por ello quitan importancia al riesgo que en todo caso conectan con el mismo proceso de progresar en investigación. Ejemplos más específicos como la experimentación en animales, se definen habitualmente como una necesidad para este progreso. Adicionalmente critican el papel de la prensa no especializada que expone erróneamente estas situaciones con un intento de vender sensacionalismo. Las personas entre vistadas exponen que ése es un tema poco importante. Sin embargo, este tema ha sido comentado por la mayoría, demostrando así un interés y un estado de opinión al respecto.

\section{Aprendiendo de los antípodas}

En sociología es útil no sólo realizar análisis longitudinales, de panel, como el anterior para el caso de los científicos e investigadores en España, sino también comparativos. Los países pueden aprender unos de otros; la sociología avanza en base a esas comparaciones. En el presente estudio se incorpora una comparación de dos realidades disimilares, pero que contienen elementos comunes interesantes: España y Australia en la actualidad. 
España puede compararse con lo que sucede en Australia25. D urante las dos últimas décadas, los investigadores y las investigadoras austral ianos se han enfrentado a cambios importantes en el sistema de investigación. D esde el G obierno existe un énfasis creciente en conectar la investigación con las necesidades nacionales, en la dirección de los fondos hacia objetivos específicos, y en el incremento de financiación privada para investigar en el sector público. La financiación se convierte en más competitiva, y existe un énfasis creciente en la responsabilidad, tanto en el proceso de decisión de distribución de recursos, como en la evaluación de la efectividad en el uso de esos recursos. D e una manera creciente también, se espera que los resultados de la investigación demuestren sus ventajas económicas y sociales, y en particular para las organizaciones en las que se realiza la investigación.

Los datos de gasto en investigación revelan el marco de colaboración y competencia de las organizaciones implicadas. En Australia, el gasto bruto en investigación y desarrollo se duplica entre los años 1984 y 1990, desde 2.400 millones de $\$ A^{26}$ hasta 5.000 millones de $\$ A$ en 199027 . Esta cantidad representa el $1,36 \%$ del PIB, comparado con el $1,25 \%$ del PIB en 198828 . En España, el gasto en I +D no llega a los 100.000 millones de ptas. en 1982, pero se incrementa hasta los 410.000 millones en $1990^{29}$. La proporción del gasto en I +D respecto al PIB aumenta desde el $0,48 \%$ en 1982 al $0,90 \%$ al final de esa década, con una tendencia de aproximación al nivel de Australia. En ambos casos, sin embargo, esas proporciones quedan lejos de los países líderes en I +D como Japón (2,98\%), Alemania (2,88\%), Estados Unidos (2,82\%), Francia $(2,34 \%)$ y Gran Bretaña $(2,25 \%)^{30}$. En Australia este crecimiento se ha duplicado en las universidades, y triplicado en el sector empresarial, aunque con un crecimiento más modesto en los institutos de investigación públicos.

A esa situación de crecimiento se le puede sumar un incremento de las relaciones entre instituciones de investigación, universidades e industria. El gobierno australiano financia un programa para el establecimiento de cincuenta centros de investigación cooperativa (cooperative research centres) en el que se implica a los tres sectores mencionados. Además, el Australian Research Council, principal organismo de financiación de la investigación básica, establece un programa de financiación para profesores einvestigadores que trabajen en colaboración con la industria. Sin embargo, se constata que las relaciones univer-

25. Véase Jesús M. DE M IGUEL (1991). « +D en sociología». Revista Española de Investigaciones Sociológi cas, 56 , p. 167-196. También Jesús M. DE M IGU EL (1992). «nvestigación de la investigación sociológica en España». Cuarto Congreso Español de Sociología. M adrid, 36 p.

26. Dólares australianos.

27. Australian Bureau of Statistics. Research and Experimental Development, All-Sector Summary, Australia, C atalogue 8112.0.

28. PIB o producto interior bruto (DG P en inglés).

29. Un análisis sectorial puede verse en Jesús M. DE M IGUEL (1989). «Funding health care: Implications for harmonization». Cross N ational Research Seminars, Londres: London School of Economics and Political Science, $27 \mathrm{p}$.

30. OECD, Science and Technology Policy, 1991 y OECD in Figures, 1992. 
sidad-empresa avanzan principalmente gracias a personas individuales. Éstas relaciones son más «apoyadas» que «conducidas» por las estrategias organizativas $^{31}$. La importancia de personas concretas en el desarrollo de esas relaciones sugiere la existencia de una cultura industrial que penetra más allá de las fronteras culturales de las universidades, y dentro de las actividades y los valores de un número creciente de investigadores universitarios. En España pare ce reflejarse un proceso parecido especialmente en los últimos años. A pesar de los esfuerzos del G obierno central y de los gobiernos autónomos para financiar y expandir el sistema de investigación, las universitarias y los universitarios necesitan buscar fuentes alternativas de financiación de la investigación en la industria. En este caso, el argumento que aparece se refleja más en el miedo a futuros recortes de la financiación pública.

La financiación creciente en base a fondos privados convierte el sistema de investigación en más competitivo. Aunque la financiación de la investigación ha aumentado durante los años ochenta y noventa, también crece la dimensión del sistema32. En ese contexto, el G obierno australiano impulsa nuevas fórmulas de financiación y establece condiciones a las instituciones, como la elaboración de planes de gestión de la investigación basados en el potencial de investigación y la capacidad de conseguir proyectos competitivos del G obierno. Los institutos de investigación y las universidades deben competir para conseguir financiar su investigación. Además, los institutos deben conseguir un $30 \%$ de sus fondos de investigación de fuentes no gubernamentales, introducir procesos sistemáticos de identificación y apoyar áreas prioritarias de investigación. D e esa manera, se establece un proceso de competencia, tanto dentro de las instituciones como entre las instituciones.

El sistema australiano parece evolucionar hacia una fórmula más competitiva. El entorno político y económico promueve más investigación en colaboración. Una característica de esa evolución se centra en la formación de los cooperative research centres (CRC), y en la investigación multidisciplinaria enfocada a la promoción de la innovación industrial. Los mecanismos de financiación de las infraestructuras en el sistema universitario promueve el uso compartido del equipamiento, y se complementa con la colaboración en proyectos promocionados a través de los proyectos en colaboración con la universidad e investigación industrial. El incremento considerable de la financiación de la investigación en el sector empresarial (un 74\% entre 1981 y 1991) da idea del crecimiento y la colaboración intersectorial. D entro de ese entorno de investigación más competitivo y de mayor colaboración, aparece una presión considerable sobre las fronteras de organización de

31. Ver, por ejemplo, Tim TURPIN y otros (1993). Crossing Innovation Boundaries The Formation and $M$ aintenance of Research Links Between Industry and U niversities in Australia, Commissioned Report N 026 , N ational Board of Employment, Education and Training. Canberra, Australia: AGPS.

32. D esde 1987, el sistema universitario australiano se ha unificado incorporando 24 universidades y 47 colleges de educación avanzada e institutos de tecnología, para formar 36 universidades del sistema nacional unificado. 
la investigación tradicionales. En ese contexto, se replantean las expectativas institucionales, las aspiraciones y las oportunidades de crecimiento, expansión o simple existencia33.

D e las entrevistas en España se desprende una evolución en el mismo sentido. Sin embargo, los mecanismos parecen menos desarrollados y la experiencia positiva de los CRC en Australia sería de posible aplicación en un futuro en España. En ambos casos las relaciones institucionales son más efectivas cuando implican la promoción de equi pos multidisciplinarios con un nivel elevado de interacción interna y externa34. Este proceso lleva a la necesidad de formación y desarrollo más planificado, una reordenación de la estrategia empresarial, y al desarrollo de un nuevo sistema de uso del conocimi ento y formas de trabajo que son todavía poco habituales en la mayoría de científicos y científicas ${ }^{35}$.

La creciente competencia y complejidad de las formas de colaboración en Australia, introduce paralelamente nuevas formas de administrar, con lo que se desarrolla la necesidad de gestionar profesionalmente la investigación. En las entrevistas de los años ochenta en España, las investigadoras y los investigadores se quejan de la introducción de las labores de gestión junto a las actividades de investigación tradicionales, y también por la falta de personal administrativo de soporte en esas tareas. En las entrevistas de principios de los noventa, los investigadores y las investigadoras se encuentran con el dilema de escoger entre la dedicación a la investigación y a las tareas de gestión. E sas al ternativas muestran conflictos entre la cultura de investigación y la cultura de docencia; pero principalmente entre estas culturas y los sistemas jerarquizados de organización de la gestión. Esta situación también se puede observar en Australia36. Sin embargo, en las antípodas el conflicto parece ser menos importante entre la autonomía de los investigadores y las investigadoras y los

33. Ello se puede observar en los planes de gestión de la investigación, los informes producidos por las universidades y los planes estratégicos de las instituciones de investigación del Gobierno durante los últimos cinco años.

34. Ver A. BLEWITT (1992). «C orporatisation and change: The reality of commercialisation for H R management in CSIRO ». Comunicación presentada en la IRR Conference, H uman Resource $M$ anagement, Reposition and Realign your H R Function for Greater Organisational Effectiveness (abril de 1992).

35. Un estudio del $\mathrm{C}$ entre for Research Policy analiza que las estrategias adoptadas por las empresas industriales para acceder a nueva tecnología y asistencia técnica son variadas. Ver CROSSIN G IN N OVATION BOUNDARIES (1993). The Formation and M aintenance of Research Links Between Industry and U niversities in Australia, Commissioned Report No 26, N ational Board of Employment, Education and Training. Canberra, Australia: AG PS. La mayor multinacional australiana, BH P, ha reestructurado recientemente su sistema de investigación, devolviendo la planificación a las divisiones. Al mismo tiempo, ha iniciado una evaluación sistemática del potencial de investigación universitario a través de visitas a la dirección de las universidades.

36. S. HILL Y T. TURPIN (1994). «The emergence of a new localism in academic research». En M arilyn Strathem (ed.). The U ses of Knowledpe: Global and Local Relations. The Reshaping of Anthropolopy, Volume 1 - Shifting C ontexts (Londres: Routledge). 
sistemas jerárquicos, y ser más un conflicto entre los criterios económicos, que juzgan el valor comercial a corto plazo, y el criterio científico e industrial, que juzga la excel encia científica y la relevancia industrial a más largo plazo37.

D esde un punto de vista comparativo, parece que las instituciones de investigación australianas son más estratégicas en la gestión de los recursos, más comerciales y creativas en la búsqueda de fórmulas que facilitan la movilidad del personal. Estos cambios se pueden observar en el C SIRO, la mayor institución investigadora en Australia. En esa institución, las nuevas iniciativas organizativas de los años ochenta incluyen los siguientes objetivos: la reducción del número de divisiones en un $25 \%$; la obtención de un $30 \%$ de financiación externa; el establecimiento de un sistema de prioridades que tengan en cuenta factores de desarrollo económico nacional y factores de tipo comercial y la reclasificación y reducción de las categorías de empleo.

El sistema de salarios y las pautas que caracterizan la nueva competencia, entran a formar parte de la estrategia de recursos humanos de la organización, con la introducción de un sistema de planificación y evaluación de todo el personal. Para los gestores de la investigación, la búsqueda de fondos para investigación representa una nueva responsabilidad que se convierte en un tema crucial en el nuevo contexto. Esos cambios forman parte de una nueva cultura organizativa. D e un modelo conducido por imperativos científicos, juicios y recompensas, se pasa a un sistema más cercano al mercado.

En España, la cultura organizativa está entrando con fuerza dentro de las instituciones. Los sistemas presupuestarios y las fórmulas de financiación aparecen en todos los niveles donde se efectúa el gasto. La búsqueda y gestión de fondos externos entra a formar parte de la política universitaria. Los mismos investigadores e investigadoras se introducen en la creciente complejidad de los sistemas de gestión de la investigación y las fórmulas de financiación públicas y privadas.

En Australia la tendencia hacia medios más organizados para dirigir y evaluar la investigación se enfrenta con cierta ambivalencia por parte de las científicas y los científicos. Por una parte existe el reconocimiento de que la investigación de los años noventa debe ser responsable de sus resultados, pero por otra parte las estructuras organizativas se viven con cierta desconfianza38. En España parece haber un reconocimiento de la necesidad de priorizar dentro de las organizaciones, así como una aceptación del papel de los gobiernos en la formulación de ese proceso. Sin embargo, también parece haber un miedo a que las áreas poco prioritarias tiendan a desaparecer. En el CSIRO, por ejemplo, los investigadores y las investigadoras opinan que el sistema está más equili-

37. T. TURPIN y A. DEVILLE (1994). «R esearch cultures and organisational change: Case studies within the CSIRO ». Informe interno para el CSIRO.

38. En 1990 el CSIRO introdujo un sistema de priorización valorando todos los programas de la institución en términos de viabilidad y capacidad de atraer a la industria. El efecto de la priorización llevó a transferir fondos desde las áreas poco prioritarias hasta las áreas altamente prioritarias. 
brado entre los objetivos científicos y los comerciales de lo que lo estaba en la década anterior. Esta diferencia de perspectivas en los dos casos analizados, puede reflejar estados diferentes de desarrollo en las relaciones entre ciencia y objetivos socioeconómicos. Pero quizás también refleja perspectivas diversas en la manera de entender la ciencia y su práctica en contextos diferentes.

En 1987 el Gobierno australiano creó el Ilamado sistema universitario unificado. Ese proceso introdujo en el mercado de la investigación a las instituciones dedicadas hasta aquel momento sólo a la docencia. El contexto investigador se traslada desde un sistema caracterizado por la autonomía individual y la decisión local sobre las prioridades, hacia un sistema de política más centralizada y procesos insti tucionales competiti vos ${ }^{39}$. Así, paral elamente a la unión de instituciones, se introduce la obligación de desarrollar planes de gestión de la investigación y se concentra el esfuerzo de investigación en nuevos grupos organizativos como centros, programas e institutos de investi gación, que se entremezclan con las fronteras de la investigación departamental más tradicionales ${ }^{40}$. En las universidades de ambos estados, durante los años ochenta se establecen oficinas de comercialización para vender sus actividades de investigación y docencia. Se establecen las oficinas de gestión de la investigación para la gestión interna, se introducen prioridades de investigación y se establecen reglas para crear, gestionar o disolver centros ${ }^{41}$. Pero en Australia se insiste en la identificación de indicadores adecuados para medir el output de investigación como una característica de los factores de calidad de la gestión de la investigación. El resultado de esas evaluaciones permite la obtención de recursos adicionales para las instituciones con bue nos resultados.

M uchas de las instituciones reciben fondos directamente del Gobierno fuera de la financiación corriente para las universidades. Por ejemplo, los cincuenta cooperative research centres, reciben un promedio de dos millones anuales de \$A del G obierno; estos fondos deben ser igualados por parte de las instituciones que participan en el centro correspondiente. Un estudio reciente demuestra que en general existe una respuesta positiva al contexto investigador y a las estrategias de gestión 42 . Los autores describen las nuevas estructuras

39. Tres de los firmantes de este artículo en el Centre for Research Policy en Australia, realizan un proyecto sobre las conexiones entre la investigación básica y los objetivos socioeconómicos. Con John Ziman (Gran Bretaña), Arie Rip (H olanda), y como consultor Daryl Chubin de la O ffice for Technolopy Assessment de Washington.

40. S. H ILL y T. TURPIN (1993). «T he clashing of academic symbols». Science as Culture, 20. Ver también «T he formation of research centres in the Australian U niversity system». Science and Technolopy Policy, 6 (5).

41. En un reciente estudio sobre universidades, el C entre for Research Policy observa que la mayoría de universidades establecen reglas para el establecimiento, gestión y disolución de centros. U no de los el ementos principales de decisión es la gestión de la investigación multidisciplinaria y las relaciones entre centros y estructuras departamentales.

42. S. LiYANAGE y H. M ITCHEL (1993). «O rganizational management in Australian cooperative research centres». Technology Analysis \& S Strategic M anagement, 5 (1). 
organizativas desde esas formas de colaboración, como «enclaves influyentes de los integrantes de investigación en colaboración». Sin embargo, esos centros crean nuevas fronteras de fidelidad y en algunos casos surgen estructuras corporativas para crear nuevas identidades y nuevas fidelidades. La pregunta más importante sigue en el aire. Se trata de averiguar hasta qué punto esos centros seguirán conectados en el futuro a los sistemas científicos o industriales en los que se formaron 43 .

Los imperativos que marcan y apoyan las nuevas estructuras en Australia reflejan la naturaleza diversa de las universidades e instituciones de investigación de los años noventa. EI CSIRO, por ejemplo, ha disuelto su empresa de comercialización de la investigación Sirotech, y ha transferido esas responsabilidades a las distintas divisiones de la organización. Paralelamente, las universidades establecen distintas formas de empresas comerciales, de propiedad o participadas por las universidades concretas. Estas empresas intervienen directamente entre las investigadoras y los investigadores académicos y el mundo comercial, que es precisamente hacia donde se dirige el esfuerzo de investigación. En una publicación titulada This Gown for $\mathrm{H}$ ire, el autor describe la forma en que los rectores de las universidades intentan solucionar el problema de integrar empre sas industriales con las actividades generales del campus. En ese proceso dan vida a la nueva profesión de managers dedicados a «motivar, mantener la motivación y encontrar y utilizar las capacidades del personal académico»44. Se refleja un conflicto de fronteras entre las redes de investigación ampliadas del científico y la científica y las redes institucionales de la universidad. El control de los fondos de investigación, la dirección de la investigación, y también el producto de la investigación entran en la discusión.

Los conflictos por el control quedan patentes en las respuestas institucionales que recibe el G obierno a partir de la iniciativa de crear una institución nacional de comercialización de la investigación Australian Technology Group (AT G ). Esa decisión se basa en una serie de medidas propuestas para mejorar la capacidad del sistema australiano de investigación y conseguir un beneficio comercial de la investigación. Es importante comparar la respuesta negativa a la propuesta por parte de las universidades y oficinas de comercialización, con la respuesta positiva de la Federación de Asociaciones de Personal U niversitario (FAU SA). La propuesta conlleva el reconocimiento de la existencia de nuevas formas estructurales de investigación, así como el reconocimiento de que necesitan ser apoyadas. La respuesta universitaria expone que AT G desafía las fronteras organizativas que mantienen las instituciones para mantener el control y el potencial de la ventaja comercial. Por parte de FAUSA,

43. Los estudios realizados en el C entre for Research Policy observan que mientras algunos CRC buscan una identidad propia, otros encuentran que su capital emerge a través de relaciones institucional es que llevan a ampliar el círculo de grupos científicos e industrial es en una red mayor.

44. P. WIN G (1993). This Gown for H ire: A H istory of the Australian Tertiary Institutions Commercial Companies Association. Canberra, Australia: Anutech. 
se reconoce que AT G puede tener el potencial de hacer más tenues las fronteras corporativas que desarrollan las universidades. En la práctica se puede decir que ATG se forma como un agente comercial y financiero complementario con el potencial de ayudar a los investigadores individuales, las universidades y las empresas.

D urante los últimos años, los institutos de investigación y las universidades responden a los nuevos imperativos y demandas de planificación, financiación y evaluación de la investigación. Se pasa hacia un modelo más comercial de organización, planificación y ejecución del trabajo de investigación. Teniendo en cuenta que sus estructuras organizativas responden a esas presiones, las investigadoras y los investigadores están en la intersección entre las estructuras y el entorno organizativos. Es un proceso a veces de legitimación, otras veces de rechazo, e incluso de modificación, pero siempre de negociación y cambio organizativo. Esta situación concuerda con lo que Alain Touraine describe como la contradicción entre acción autónoma por la parte del personal, y la dirección y las restricciones que ejercen las organizaciones para conseguir sus objetivos corporativos ${ }^{45}$. En el model o de Touraine, las crisis de la acción son el producto de las contradicciones inherentes entre restricción y autonomía, emplazando el análisis en el poder y el control organizativo, más que en la definición o redefinición de objetivos organizativos ${ }^{46}$. Las agencias de investigación australianas experimentan tensiones a causa de los conflictos por el control de la acción, más que por las direcciones de la acción como característica de las tensiones de los años ochenta.

Estos conflictos caracterizan también a las investigadoras y los investigadores e instituciones de investigación en E spaña. La aprobación de la LRU, la Ley de la Ciencia, y las iniciativas de política científica promovidas por el Gobierno central y el gobierno de la Generalitat de Catalunya, reflejan una cierta competición por el control de la investigación 47 . Sin embargo, no son sólo conflictos entre niveles diferentes de gobierno, sino también entre formas distintas de dirigir y juzgar la ciencia, así como los valores inherentes a los principios y prácticas de gestionar las organizaciones gubernamentales y empresariales en los años noventa. A ello se le unen problemas de estabilidad en la aplicación de las medidas de política de investigación 48 .

La evolución de la formación para la investigación en las universidades australianas en los años noventa, es paral ela al cambio del sistema de investiga-

45. A. Touraine (1971). The Post-industrial Society. Nueva York: Random H ouse.

46. Véase también Jesús M. DE M Iguel (1991). El mito de la sociedad organizada. Barcelona: Península.

47. Ver CICYT (1988). Plan N acional de organización cientifica y desarrollo tecnológico. M adrid: M EC; ver también CIRIT (1988). Programes de recerca i desenvolapament de la Generalitat de Catalunya: 1988-1992. Barcelona: D epartament de Presidència de la G eneralitat de Catalunya.

48. L. Sanz-M en Éndez, E. M uñoz y C.E. Garcí (1993). «Rise and fall. The vicissitudes of Spanish science and technology policy: Coordination and leadership». Science and Public Policy, 20 (6), p. 370-380. 
ción. La enseñanza universitaria se encuentra en un número superior de instituciones y en un marco más orientado hacia el mercado. Existe una demanda creciente de programas doctorales dedicados a áreas de conocimiento industrial. Al mismo tiempo, la concentración de la investigación en las universidades se aleja de las estructuras departamentales tradicionales, y se organizan en estructuras de centro con un enfoque multidisciplinario. Para los grupos consolidados durante los años noventa se mantiene el valor de servir como una fuente creciente de formación para la investigación, así como una cantera de empleo para las estudiantes y los estudiantes graduados. En la evolución de ese proceso se espera que aparezca una demanda mayor de coordinación de las prácticas de supervisión de estudiantes de investigación y calidad de la formación. Esa tendencia puede provocar al mismo tiempo una separación entre enseñanza e investigación universitarias.

D urante los años ochenta se observa un aumento del apoyo financiero a los investigadores y las investigadoras en formación. La tendencia cambia desde el apoyo directo al investigador que se está formando, hacia una política de atracción de los mejores estudiantes para seguir una carrera investigadora. De manera creciente los centros de investigación en las universidades facilitan financiación adicional a las becas del Gobierno para hacerlas más interesantes para promocionar la formación de investigadores. El enfoque principal de la formación para la investigación durante los años noventa se dirige al desarrollo curricular, al establecimiento de puentes con la industria y a atraer los estudiantes y las estudiantes más apropiados, más que a seguir una política de números departamentales. La calidad y las discusiones acerca de las prioridades nacionales e institucional es están por encima de las demandas de distribución equitativa de recursos entre disciplinas e instituciones. Esta situación contrasta con el caso de España, donde a pesar de la crítica a la formación recibida tanto por su calidad como por la falta de medios infraestructurales, no se entra en profundidad en la discusión estructural de los mecanismos de asignación institucional. Se centra en la mecánica propia del grupo y su formación mediante el desarrollo de proyectos de investigación.

Una característica del contexto investigador en la Australia de los años noventa es el desarrollo de indicadores para el análisis de los resultados de la investigación y la calidad de la gestión. Los últimos cambios en el sistema universitario conllevan una discusión creciente acerca de la calidad del sistema universitario. Esta discusión se centra en la gestión del crecimiento y mantenimiento de la equidad en la participación, y en mantener una calidad dentro de la creciente diversidad de los programas de estudio. La mayoría de las instituciones desarrollan normas de funcionamiento para evaluar y mantener la calidad académica universitaria. El Comité de Rectores (AVCC) y el C onsejo de Asociaciones de Postgrados publican unas normas para el mantenimiento del nivel de calidad, las cuales son adoptadas por la mayoría de universidades australianas.

Un tema clave que aparece en las publicaciones gubernamentales sobre la calidad es el énfasis en el mantenimiento de la autonomía universitaria. Sin embargo, se observa un equilibrio entre autonomía y responsabilidad, sobre 
todo a través del medio de la gestión de la calidad para el sistema como un todo, y la garantía de la calidad como un principio clave del desarrollo. U na publicación reciente 49 hace referencia específica al desarrollo de una estructura nacional que garantice la calidad. A pesar de que los conceptos de Total Q uality $M$ anagementy, Q uality Assurance, son recientes en el discurso académico, van incorporándose poco a poco a la práctica general. Parece que la necesidad de mecanismos que faciliten la sinergia entre autonomía y responsabilidad llevarán a alguna nueva fórmula de gestión total de la calidad adecuada dentro del sistema.

Tanto en España como en Australia se observa una tendencia hacia la evaluación externa y objetiva de los resultados de la investigación. Es importante destacar que en España el énfasis es en evaluación individual, mientras que en Australia es institucional. El debate sobre la calidad en Australia lleva a un interés creciente por el establecimiento de indicadores que se puedan usar para la distribución de «recompensas» financieras a las instituciones que consiguen los mejores resultados, teniendo en cuenta unos indicadores determinados ${ }^{50}$.

En Australia se han llevado a cabo dos estudios recientes para establecer los indicadores preferidos por los investigadores y las investigadoras para juzgar el valor de una investigación realizada51. Los investigadores y las investigadoras universitarios enfatizan los indicadores asociados con los outputs de publicación, impacto y participación en congresos importantes, que se asemejan a los de las entrevistas de la década de los noventa realizadas en la U niversitat de Barcel ona. Los investigadores y las investigadoras del CSIRO, sin embargo, aunque escojan el output de publicación como el primer indicador, prefieren ser evaluados también por la relevancia industrial de sus resultados, medida a través de contratos de investigación y la producción de productos patentables y de procesos. Estas diferencias muestran la distinción entre localizaciones organizativas diferentes en el trabajo de las investigadoras y los investigadores. También enseñan la diferencia de objetivos. Estas perspectivas demuestran hasta qué punto la investigación australiana aparece cada vez más diferenciada a causa de fronteras organizativas. La respuesta de las investiga-

49. H igher Education Council, N ational Board of Employment, Education and Training, Higher Education: Achieving Q uality (AG PS, octubre de 1992).

50. Por ejemplo, la financiación de la infraestructura de investigación en las universidades australianas se distribuye teniendo en cuenta la cantidad de financiación conseguida por cada universidad en financiación previa de proyectos. Para una evaluación institucional en Cataluña, ver Joan BELLAVISTA y otros (1993). Política científica y tecnológica: evaluación de la I+D en la Universitat de Barcelona. Barcelona: Publicacions de la Universitat de Barcelona; también en C. Viladiu, L. Escriban O, J. Bellavista, M. Grabulós, E. Guardiola, C. I glesias y D. SerRat. «A research evaluation model of a large, ancient University», Research Evaluation, 2 (3) (1992), p. 124-134.

51. Ver L. GRIG y P. SheEHAN (1993). Research Performance Indicators Survey, N BEET, Commissioned Report N 0. 21. Canberra, Australia: Australian Government Publishing Service. Ver también T. TURPIN, A. D EVILLE y otros (1993). Crossing Innovation Boundaries: The Formation and $M$ aintenance of Research LinksB Between Industry and U niversities in Australia, N BEET Commissioned Report N 0. 26. Canberra, Australia: AG PS. 
doras y los investigadores entrevistados en Barcel ona a ser evaluados, es comparable a la que se observa en Australia. Sin embargo, las presiones del sistema de investigación en España para priorizar, evaluar y depender de los resultados de la investigación parecen ser menos importantes. Las fronteras organizativas parecen también desarrollarse en el contexto del sistema de investigación actual.

Tanto en España como en Australia, la investigación se ha ido sistematizando. Las instituciones se convierten en más competitivas, más orientadas en lo comercial, más estratégicas, introduciendo prácticas y valores de gestión. Al mismo tiempo, esos cambios estructurales llevan a tensiones entre las estructuras del pasado - los departamentos académicos y las unidades de investigación basadas en disciplinas científicas- y las nuevas estructuras de investigación en forma de centros de investigación y redes de cooperación investigadora. Aunque los recursos públicos son menos esenciales, los gobiernos piden una mayor responsabilidad en el gasto. La política de investigación elige como objetivo principal las fronteras entre sectores y entre disciplinas. Parece que ese objetivo está bien definido, a tenor de la angustia que muestran los cancerberos culturales. Las fronteras entre investigación y sociedad son también un objetivo claro. La ética en el trabajo y sus resultados, las limitaciones y controles sobre el uso de animales de experimentación y la ética humana, son temas de los años noventa. Pero queda un largo proceso de introducción a la cultura de los investigadores y las investigadoras. U na serie de universidades australianas han sido penalizadas financieramente por la falta de esta blecimiento de indicadores definidos para asegurar la calidad en sus relaciones con el bienestar de la comunidad.

En el presente artículo sociológico se ha analizado la investigación de las décadas de los ochenta y noventa con sus características de conflictos entre fronteras. El resultado de esas fronteras puede producir sistemas de investigación que tienen una potencia cultural considerable. Sin embargo, es posible continuar reconstruyendo esos artefactos. Pero para el futuro es importante que se investigue la comprensión del proceso en el cual ocurre, y que se ofrezca la posibilidad de un proceso de aprendizaje sobre la operatividad de las políticas a seguir. La sociología es un buen instrumento para analizar esos cambios dentro de la ciencia y en las sociedades 52 . Incluso de los antípodas.

52. Jesús M. De M iguel, y M elissa G. M oyer (1979). Sociology in Spain (Beverly H ills, California: Sage Publications, 299 p. 